\title{
Comparison of Efficacy and Safety of Febuxostat in Gout Patients with Chronic Kidney Disease Stage 3 and Stage 4/5
}

\author{
Eeunyoung Ahn ${ }^{1}$, Sunggun Lee ${ }^{2}$, Han-Na Lee ${ }^{3}$, Seung-Geun Lee ${ }^{3}$, Min Wook So ${ }^{1}$ \\ ${ }^{1}$ Division of Rheumatology, Department of Internal Medicine, Pusan National University Yangsan Hospital, Yangsan, ${ }^{2}$ Division of Rheumatology, \\ Department of Internal Medicine, Haeundae Paik Hospital, Inje University College of Medicine, Busan, ${ }^{3}$ Division of Rheumatology, Department \\ of Internal Medicine, Pusan National University Hospital, Busan, Korea
}

\begin{abstract}
Objective. To compare efficacy and safety of febuxostat in gouty patients with chronic kidney disease (CKD) stage 3 and stage 4/5. Methods. Age and sex matched patients with CKD stage 3 and stage $4 / 5$ who were diagnosed with gout were included. The dose of febuxostat was increased according to serum uric acid (sUA) level. Adherence, the number of gout attack, the change of sUA, the change of estimated glomerular filtration rate (eGFR) and adverse events (AEs) were evaluated for 12 months. Results. There were no significant differences in the baseline variables between CKD stage 3 and CKD stage 4/5. Disease duration was longer and baseline sUA was higher in the CKD stage $4 / 5$. There were no significant differences in the mean sUA at the last follow-up, the number of patients who reached the sUA target of $6 \mathrm{mg} / \mathrm{dL}$ and the number of gout attack between the groups. There were no significant differences in the change of eGFR and decrease of eGFR between the groups. There were 2 cases of AEs. One patient in CKD stage 3 had maculopapular rash and one patient in CKD stage 4/5 had dizziness. The AEs were subsided after febuxostat was stopped. Conclusion. Febuxostat was efficacious and well tolerated in gout patients with CKD stage 4/5. (J Rheum Dis 2019;26:118-123)
\end{abstract}

Key Words. Gout, Febuxostat, Chronic kidney disease

\section{INTRODUCTION}

Gout is a chronic disease caused by the precipitation of monosodium urate crystals in the joints, leading to inflammation, pain and joint destruction [1]. Chronic kidney disease $(\mathrm{CKD})$ is responsible for hyperuricemia and is one of the major risk factors of gout. A previous study estimated the prevalence of CKD in patients with gout to be $39 \%$ [2].

Urate-lowering therapies (ULT) are initiated in chronic gout in order to maintain normal serum uric acid (sUA) levels and to prevent further attacks $[3,4]$. The European League Against Rheumatism recommended allopurinol as the first line ULT and daily dose restrictions in CKD patients depending on creatinine clearance values [5]. The recommended allopurinol dose for patients with $\mathrm{CKD}$ stage $4 / 5$ is usually too low to reach the therapeutic sUA target $[6,7]$. Febuxostat, a non-purine selective xanthine oxidase inhibitor which is predominantly metabolized in the liver, can be used for patients with CKD stage 1 3 without dose adaptation $[8,9]$. However, efficacy and safety of febuxostat in patients with CKD stage $4 / 5$ have not been established due to the lack of evidence in this population $[5,10]$. Although a few studies have investigated efficacy and safety of febuxostat in severe CKD, most of them were retrospective or single arm design studies [11-14].

Herein, we performed a prospective observational study to compare efficacy and safety of febuxostat in gouty patients with CKD stage 3 and stage 4/5.

\footnotetext{
Received : November 23, 2018, Revised : (1st) December 7, 2018, (2nd) December 13, 2018, Accepted : December 14, 2018

Corresponding to : Min Wook So (iD http://orcid.org/0000-0001-5027-0410

Division of Rheumatology, Department of Internal Medicine, Pusan National University Yangsan Hospital, 20 Geumo-ro, Mulgeum-eup, Yangsan 50612, Korea. E-mail : thalsdnrso@naver.com
}

Copyright (c) 2019 by The Korean College of Rheumatology. All rights reserved.

This is an Open Access article, which permits unrestricted non-commerical use, distribution, and reproduction in any medium, provided the original work is properly cited. 


\section{MATERIALS AND METHODS}

\section{Study design and patients}

This prospective observational cohort study was conducted in the rheumatology center of a local tertiary hospital in Korea. Age and sex matched 48 patients with CKD stage 3 and 48 patients with CKD stage $4 / 5$ who were diagnosed with gout were included [15]. Of the 48 patients with CKD stage 4/5, 12 patients were on hemodialysis. We excluded patients with chronic liver disease due to concerning for hepatotoxicity. This study was approved by the regional Institutional Review Board and informed consent was obtained from all patients. CKD stage 3 was defined as estimated glomerular filtration rate (eGFR) $<60$ and $\geq 30 \mathrm{~mL} / \mathrm{min} / 1.73 \mathrm{~m}^{2}$ and CKD stage $4 / 5$ was defined as eGFR $<30 \mathrm{~mL} / \mathrm{min} / 1.73 \mathrm{~m}^{2}$ for at least 3 months before febuxostat initiation [16]. All patients were ULT naïve or allopurinol resistant cases. Total study duration was 12 months. Treatment with febuxostat was initiated at $40 \mathrm{mg}$ per day and the dose was increased according to sUA level. Either low dose colchicine or low dose prednisolone was prescribed for all patients to prevent acute flare during 6 months. All patients except patients on hemodialysis were prescribed colchicine $0.3 \mathrm{mg}$ per day and patients on hemodialysis were prescribed prednisolone 5 mg per day. Adherence was evaluated during the study period. Adherence to febuxostat was assessed by the clinic nurses through pill counts at the scheduled visits to the outpatient clinic and non-adherence was defined as taking less than $80 \%$ of the prescribed medication. Non-adherent cases were excluded from the outcome analysis.

\section{Variables}

Demographic factors (age and gender), smoking status, alcohol drinking status and specific comorbidities (hypertension, diabetes mellitus, cardiac disease, history of malignancy and diuretics use) were examined. Current smoker was defined as a person who reported to smoke someday or every day or who reported abstinence from cigarettes less than one year. Alcohol drinker was defined as a person who reported drinking more than one drink per week. Cardiac disease was defined as a major cardiac condition including coronary artery disease (angina pectoris or myocardial infarction), heart failure, valvular heart disease, or any type of symptomatic arrhythmia. Moreover, factors associated with gout (disease duration, switch from allopurinol and baseline sUA level) and the baseline eGFR were also examined. sUA level, eGFR, fre- quencies of acute gouty attack and adverse events (AEs) related to febuxostat were checked at the scheduled visits during the 12 months of observation.

\section{Statistical analysis}

The means and standard deviations (SD) and percentages were used to describe the clinical characteristics of the participants. T-test (or Mann-Whitney U-test) and chi-square analysis were used to measure differences between the groups. Dropout, non-adherent cases and patients who stopped febuxostat due to AEs were included in the baseline but not in the outcome analysis. Variables with p-values $<0.05$ was considered statistically significant. All statistical calculations were performed using IBM SPSS version 21 (IBM Co., Armonk, NY, USA).

\section{RESULTS}

Age and sex matched 48 patients with CKD stage 3 and 48 patients with CKD stage $4 / 5$ were included. Eleven patients from the CKD stage 3 and 13 from CKD stage 4/5 were either dropout, non-adherent or AEs cases. Among 48 patients of each group, 37 patients in CKD stage 3 and 35 patients in CKD stage $4 / 5$ were included in the outcome analysis. Of the 35 patients with CKD stage 4/5, 9 patients were on hemodialysis (Figure 1). The baseline characteristics of the patients are presented in Table 1. The mean age of the patients was $66.9 \pm 12.7$ and $67.1 \pm 12.6$ years, respectively, and the majority of the patients were male $(75.0 \%)$. There were no significant differences in the demographic factors (age and gender), smoking status, alcohol drinking status and specific comorbidities between the groups. In gout characteristics,

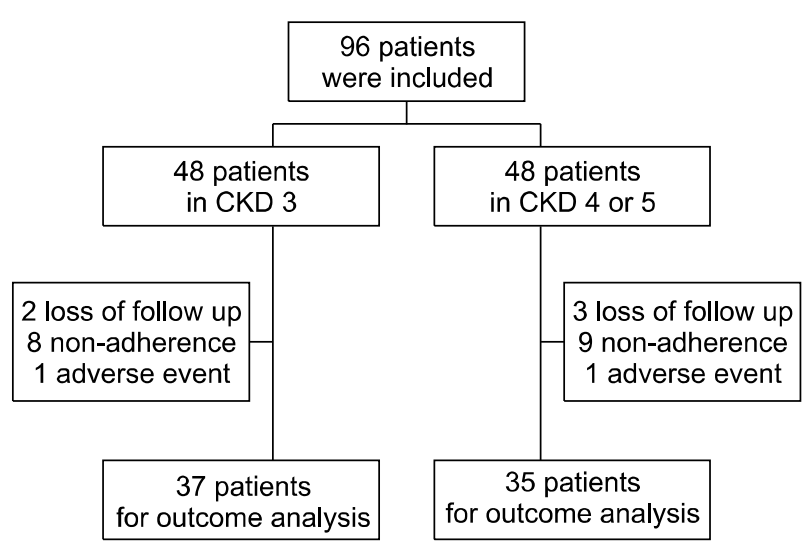

Figure 1. Flow of the patients through the trial. CKD: chronic kidney disease. 
Eeunyoung Ahn et al.

Table 1. Baseline characteristics of the patients

\begin{tabular}{|c|c|c|c|}
\hline Characteristics & CKD $3(n=48)$ & CKD 4/5 (n=48) & p-value \\
\hline Age $(y r)$ & $66.9 \pm 12.7$ & $67.1 \pm 12.6$ & 0.923 \\
\hline Gender, male & $36(75.0)$ & $36(75.0)$ & 1.000 \\
\hline Smoking, current & $2(4.2)$ & $4(8.3)$ & 0.677 \\
\hline Alcohol drinking & $12(25.0)$ & $10(20.8)$ & 0.809 \\
\hline \multicolumn{4}{|l|}{ Comorbidities } \\
\hline Hypertension & $38(79.2)$ & $44(91.7)$ & 0.146 \\
\hline Diabetes mellitus & $12(25.0)$ & $4(8.3)$ & 0.053 \\
\hline Cardiac disease & $26(54.2)$ & $18(37.5)$ & 0.151 \\
\hline History of malignancy & $6(12.5)$ & $6(12.5)$ & 1.000 \\
\hline Diuretics use & $14(29.2)$ & $24(50.0)$ & 0.060 \\
\hline Aspartate aminotransferase (IU/L) & $30 \pm 8.4$ & $32 \pm 8.2$ & 0.902 \\
\hline Alanine aminotransferase (IU/L) & $27 \pm 7.2$ & $29 \pm 8.0$ & 0.819 \\
\hline \multicolumn{4}{|l|}{ Gout characteristics } \\
\hline Disease duration (mo) & $39.5 \pm 51.8$ & $84.0 \pm 95.4$ & 0.008 \\
\hline Switch from allopurinol & $12(25.0)$ & $6(12.5)$ & 0.190 \\
\hline Baseline sUA (mg/dL) & $8.9 \pm 1.8$ & $9.8 \pm 2.7$ & 0.049 \\
\hline \multicolumn{4}{|l|}{ Renal characteristics } \\
\hline Baseline eGFR $\left(\mathrm{mL} / \mathrm{min} / 1.73 \mathrm{~m}^{2}\right)$ & $46.3 \pm 9.0$ & $18.3 \pm 7.7$ & 0.000 \\
\hline
\end{tabular}

Values are presented as mean \pm standard deviation or number (\%). CKD: chronic kidney disease, sUA: serum uric acid, eGFR: estimated glomerular filtration rate.

Table 2. Outcomes analysis of the patients

\begin{tabular}{lccc}
\hline \multicolumn{1}{c}{ Outcomes } & CKD 3 $(\mathrm{n}=37)$ & CKD 4/5 $(\mathrm{n}=35)$ & $\mathrm{p}$-value \\
\hline Gout characteristics & & & \\
$\quad$ Final dose of febuxostat $(\mathrm{mg} / \mathrm{dL})$ & $46.5 \pm 14.9$ & $42.3 \pm 9.4$ & 0.159 \\
$\quad$ Number of gout attack & $0.9 \pm 1.0$ & $0.7 \pm 0.8$ & 0.719 \\
$\quad$ Final sUA $(\mathrm{mg} / \mathrm{dL})$ & $4.9 \pm 1.3$ & $5.0 \pm 2.1$ & 0.942 \\
$\quad$ Final sUA $<6.0 \mathrm{mg} / \mathrm{dL}$ & $28(75.7)$ & $28(80.0)$ & 0.779 \\
Renal characteristics & & & $0.0 \pm 5.8$ \\
$\quad$ Change of eGFR $\left(\mathrm{mL} / \mathrm{min} / 1.73 \mathrm{~m}^{2}\right)$ & $19(51.4)$ & $23(65.7)$ & 0.649 \\
$\quad$ Decrease of eGFR & $1(2.6)$ & $1(2.8)$ & 0.241 \\
Adverse event & & 1.000 \\
\hline
\end{tabular}

Values are presented as mean \pm standard deviation or number (\%). CKD: chronic kidney disease, sUA: serum uric acid, eGFR: estimated glomerular filtration rate.

disease duration of gout was longer $(39.5 \pm 51.8$ vs. $84.0 \pm 95.4$ months, $\mathrm{p}=0.008)$ and the baseline sUA was higher $(8.9 \pm 1.8$ vs. $9.8 \pm 2.7 \mathrm{mg} / \mathrm{dL}, \mathrm{p}=0.049)$ in the CKD stage 4/5 (Table 1).

In the outcome analysis, there were no significant differences in the mean sUA at the last follow-up $(4.9 \pm 1.3 \mathrm{vs}$. $5.0 \pm 2.1 \mathrm{mg} / \mathrm{dL})$, the number of patients who reached the sUA target of $6 \mathrm{mg} / \mathrm{dL}$ (75.7\% vs. $80.0 \%)$ and the number of gout attack $(0.9 \pm 1.0$ vs. $0.7 \pm 0.8)$ between the groups. Moreover, there were no significant differences in the final dose of febuxostat $(46.5 \pm 14.9$ vs. $42.3 \pm 9.4 \mathrm{mg} / \mathrm{dL})$ between the groups. In renal characteristic, there were no significant differences in the change of eGFR and decrease of eGFR between the groups (Table 2). There were 2 cases of AEs. A 79 year old female with CKD stage 3 had maculopapular rash 2 weeks after the initiation of febuxostat and was subsided after febuxostat was stopped. Another 77 year old male patient who was on hemodialysis due to CKD stage 5 had dizziness one month after the initiation of febuxostat and was subsided after febuxostat was stopped.

After exclusion of patients on hemodialysis, the results were also similar (Table 3 ). There were no significant differences in the mean sUA at the last follow-up $(4.9 \pm 1.3$ 
Table 3. Outcomes analysis of the patients (after exclusion of patients on hemodialysis)

\begin{tabular}{lccc}
\hline \multicolumn{1}{c}{ Outcomes } & CKD 3 $(\mathrm{n}=37)$ & CKD 4/5 $(\mathrm{n}=26)$ & $\mathrm{p}$-value \\
\hline \hline Gout characteristics & & & \\
Final dose of febuxostat $(\mathrm{mg} / \mathrm{dL})$ & $46.5 \pm 14.9$ & $43.1 \pm 10.9$ & 0.299 \\
$\quad$ Number of gout attack & $0.9 \pm 1.0$ & $0.6 \pm 0.8$ & 0.264 \\
Final sUA $(\mathrm{mg} / \mathrm{dL})$ & $4.9 \pm 1.3$ & $4.4 \pm 1.5$ & 0.157 \\
Final sUA $<6.0 \mathrm{mg} / \mathrm{dL}$ & $28(75.7)$ & $22(84.6)$ & 0.531 \\
Renal characteristics & & & 0.988 \\
Change of eGFR $\left(\mathrm{mL} / \mathrm{min} / 1.73 \mathrm{~m}^{2}\right)$ & $-0.6 \pm 4.8$ & $-0.6 \pm 6.5$ & 0.452 \\
Decrease of eGFR & $19(51.4)$ & $16(61.5)$ & 1.000 \\
Adverse event & $1(2.6)$ & $0(0.0)$ & \\
\hline
\end{tabular}

Values are presented as mean \pm standard deviation or number (\%). CKD: chronic kidney disease, sUA: serum uric acid, eGFR: estimated glomerular filtration rate.

vs. $5.0 \pm 2.1 \mathrm{mg} / \mathrm{dL})$, the number of patients who reached the sUA target of $6 \mathrm{mg} / \mathrm{dL}(75.7 \%$ vs. $80.0 \%)$, the number of gout attack $(0.9 \pm 1.0$ vs. $0.7 \pm 0.8)$ and the final dose of febuxostat $(46.5 \pm 14.9$ vs. $42.3 \pm 9.4 \mathrm{mg} / \mathrm{dL}$ ) between the groups. Moreover, there were no significant differences in the change of eGFR and decrease of eGFR between the groups.

\section{DISCUSSION}

Febuxostat, by its hepatic metabolism, is useful for CKD patients, but its use for stage $4 / 5 \mathrm{CKD}$ patients has not been recommended due to lack of data from phase 3 trials in this population. Although a few studies have investigated efficacy and safety of febuxostat in CKD stage 4/5, these studies had some limitations [11-14]. Thus, this study was conducted to evaluate and compare efficacy and safety of febuxostat in gouty patients with CKD stage 3 and stage $4 / 5$. The study results showed that there were no significant differences in the mean sUA at the last follow-up, the number of patients who reached the sUA target of $6 \mathrm{mg} / \mathrm{dL}$ and the number of gout attack between the groups. A previous study conducted with 73 gouty patients with stage $4 / 5 \mathrm{CKD}$ also reported similar results. The mean sUA level at last follow-up was $5.0 \pm 2.3 \mathrm{mg} / \mathrm{dL}$ and $65.1 \%$ of the patients reached the sUA target of 6 $\mathrm{mg} / \mathrm{dL}$ [11]. Another study conducted with gouty patients with moderate to severe renal impairment (CKD stage $3 / 4$ ) reported that the proportion of patients with sUA level of $<6.0 \mathrm{mg} / \mathrm{dL}$ at month 12 was greater in various febuxostat dosage group than in the placebo group (45.2\% 68.8\% vs. 0\%) [17].

In renal characteristic, $51.4 \%$ of CKD stage 3 and $65.7 \%$ of CKD stage $4 / 5$ patients showed decrease of eGFR.
However, the changes of eGFR were minimal in both groups. Thus, there were no significant differences in the change of eGFR and decrease of eGFR between the groups in our study. The results were also similar after exclusion of patients on hemodialysis. Because of our study design and difference of the baseline renal status, no firm conclusion can be made about the impact of febuxostat on renal function. Thus, we only reported that there were no significant differences in the change of eGFR and decrease of eGFR between the groups. Febuxostat was suggested to improve renal function by lowering sUA level but results are still controversial $[17,18]$. One study reported that the intake of febuxostat led to slow the decline in eGFR in CKD stage 3/4 compared to placebo [18] while another study reported that the differences in serum creatinine level and eGFR were not statistically significant between febuxostat and placebo group [17]. One large scale randomized controlled trial (FEATHER trail) is currently underway to resolve this controversial.

In our study, there were only 2 cases of AEs. One patient in CKD stage 3 had maculopapular rash and one patient in CKD stage $4 / 5$ had dizziness. This difference was not significant between the groups ( $2.6 \%$ vs. $2.8 \%$ ). There were no serious AEs. Especially, despite a large number of patients with cardiac disease in our study (46\%), no cardiovascular event was reported. This result is consistent with results of other studies [11,18]. A previous study conducted with 73 gouty patients with stage $4 / 5$ CKD reported only a case of limb edema as an adverse event and there were no serious AEs [11]. Another study conducted with CKD stage $3 / 4$ patients with asymptomatic hyperuricemia reported that only 2 patients had mild diarrhea [18].

This study has some limitations. First, it was conducted 
as a single center study with a small sample size and short follow-up duration. Especially, the number of patients in the trial may have been insufficient to assess safety. Thus, the findings need to be corroborated over multi-center studies with larger sample sizes and longer follow-up durations. Second, $25 \%$ of the total patients were excluded in the outcome analysis because of various reasons (dropout, non-adherence or AEs) and this limitation weakens the merits of the study design. However, most of the exclusion cases were non-adherent patients (70\%). A previous study conducted with 132 gouty patients in a routine clinical setting, adherence (compliance) of ULT was $71.2 \%$. This rate was similar in our study [19]. Some of previous studies reporting efficacy and safety of ULT in CKD was not evaluated the adherence. Without information about adherence, efficacy may have been underestimated and safety may have been overestimated. In our study, adherence to febuxostat was exactly assessed by the clinic nurses through pill counts at the scheduled visits to the outpatient clinic and non-adherent cases were excluded in the outcome analysis. Finally, even though we included patients on hemodialysis, sample size was too small (12 patients were included and 9 patients was completed the study). Therefore, it was difficult to assess detailed information about efficacy and safety in patients on hemodialysis. Despite some limitations, this study was conducted with prospective observational cohort design. This is the strength of our study.

\section{CONCLUSION}

Our study results showed that febuxostat is efficacious and well tolerated in gout patients with CKD stage $4 / 5$ compared to CKD stage 3.

\section{ACKNOWLEDGMENTS}

This study was supported by a 2018 research grant from Pusan National University Yangsan Hospital. We would like to acknowledge the invaluable collaboration of the rheumatology center nursing team of Pusan National University Yangsan Hospital.

\section{CONFLICT OF INTEREST}

No potential conflict of interest relevant to this article was reported.

\section{REFERENCES}

1. Schlesinger N. Difficult-to-treat gouty arthritis: a disease warranting better management. Drugs 2011;71:1413-39.

2. Fuldeore MJ, Riedel AA, Zarotsky V, Pandya BJ, Dabbous O, Krishnan E. Chronic kidney disease in gout in a managed care setting. BMC Nephrol 2011;12:36.

3. McClory J, Said N. Gout in women. Med Health R I 2009;92:363-4, 368.

4. Shoji A, Yamanaka H, Kamatani N. A retrospective study of the relationship between serum urate level and recurrent attacks of gouty arthritis: evidence for reduction of recurrent gouty arthritis with antihyperuricemic therapy. Arthritis Rheum 2004;51:321-5.

5. Richette P, Doherty M, Pascual E, Barskova V, Becce F, Castañeda-Sanabria J, et al. 2016 updated EULAR evidence-based recommendations for the management of gout. Ann Rheum Dis 2017;76:29-42.

6. Richette P, Frazier A, Bardin T. Pharmacokinetics considerations for gout treatments. Expert Opin Drug Metab Toxicol 2014;10:949-57.

7. Dalbeth N, Kumar S, Stamp L, Gow P. Dose adjustment of allopurinol according to creatinine clearance does not provide adequate control of hyperuricemia in patients with gout. J Rheumatol 2006;33:1646-50.

8. Mukoyoshi M, Nishimura S, Hoshide S, Umeda S, Kanou M, Taniguchi $\mathrm{K}$, et al. In vitro drug-drug interaction studies with Febuxostat, a novel non-purine selective inhibitor of xanthine oxidase: plasma protein binding, identification of metabolic enzymes and cytochrome P450 inhibition. Xenobiotica 2008;38:496-510.

9. Hira D, Chisaki Y, Noda S, Araki H, Uzu T, Maegawa H, et al. Population pharmacokinetics and therapeutic efficacy of Febuxostat in patients with severe renal impairment. Pharmacology 2015;96:90-8.

10. Schumacher HR Jr, Becker MA, Wortmann RL, Macdonald PA, Hunt B, Streit J, et al. Effects of febuxostat versus allopurinol and placebo in reducing serum urate in subjects with hyperuricemia and gout: a 28-week, phase III, randomized, double-blind, parallel-group trial. Arthritis Rheum 2008;59:1540-8.

11. Juge PA, Truchetet ME, Pillebout E, Ottaviani S, Vigneau C, Loustau C, et al. Efficacy and safety of febuxostat in 73 gouty patients with stage 4/5 chronic kidney disease: a retrospective study of 10 centers. Joint Bone Spine 2017;84:595-8.

12. Quilis N, Andrés M, Gil S, Ranieri L, Vela P, Pascual E. Febuxostat for patients with gout and severe chronic kidney disease: which is the appropriate dosage? Comment on the article by Saag et al. Arthritis Rheumatol 2016;68:2563-4.

13. Lim DH, Oh JS, Ahn SM, Hong S, Kim YG, Lee CK, et al. Febuxostat in hyperuricemic patients with advanced CKD. Am J Kidney Dis 2016;68:819-21.

14. Sakai Y, Otsuka T, Ohno D, Murasawa T, Sato N, Tsuruoka S. Febuxostat for treating allopurinol-resistant hyperuricemia in patients with chronic kidney disease. Ren Fail 2014;36:225-31.

15. Wallace SL, Robinson H, Masi AT, Decker JL, McCarty DJ, Yü TF. Preliminary criteria for the classification of the acute arthritis of primary gout. Arthritis Rheum 1977;20:895-900.

16. Levey AS, Bosch JP, Lewis JB, Greene T, Rogers N, Roth D. 
A more accurate method to estimate glomerular filtration rate from serum creatinine: a new prediction equation. Modification of Diet in Renal Disease Study Group. Ann Intern Med 1999;130:461-70.

17. Saag KG, Whelton A, Becker MA, MacDonald P, Hunt B, Gunawardhana L. Impact of Febuxostat on renal function in gout patients with moderate-to-severe renal impairment. Arthritis Rheumatol 2016;68:2035-43.
18. Sircar D, Chatterjee S, Waikhom R, Golay V, Raychaudhury A, Chatterjee S, et al. Efficacy of Febuxostat for slowing the GFR decline in patients with CKD and asymptomatic hyperuricemia: a 6-month, double-blind, randomized, placebo-controlled trial. Am J Kidney Dis 2015;66:945-50.

19. Lee S, So MW. Adherence with urate-lowering therapies among male patients with gout in a routine clinical setting. Mod Rheumatol 2016;26:950-5. 